\title{
Lapurdum
}

LAPURDUM Euskal ikerketen aldizkaria | Revue d'études basques |

Revista de estudios vascos | Basque studies review

$3 \mid 1998$

Numéro III

\section{Art poétique basque Adéma « Zalduby » rena}

\section{Xabier Altzibar}

\section{OpenEdition}

\section{Journals}

Édition électronique

URL : https://journals.openedition.org/lapurdum/1684

DOI : 10.4000/lapurdum. 1684

ISSN : 1965-0655

Éditeur

IKER

Édition imprimée

Date de publication : 1 octobre 1998

Pagination : $77-101$

ISBN : 2-84127-152-8

ISSN : $1273-3830$

\section{Référence électronique}

Xabier Altzibar, « Art poétique basque Adéma « Zalduby » rena », Lapurdum [En ligne], 3 | 1998, mis en ligne le 01 septembre 2010, consulté le 24 février 2022. URL : http://journals.openedition.org/ lapurdum/1684; DOI : https://doi.org/10.4000/lapurdum.1684 


\section{Xabier ALTZIBAR}

\section{ART POÉTIQUE BASQUE ADÉMA "ZALDUBY" RENA}

Gratien Adéma "Zalduby" (1828-1907) poetaren Ian hau Eskualduna kazetan argitara zen 1899 . urtean, zatika, data hauetako agerraldietan (alea, urtea, hilabetea, eguna hurrenez-hurren) : 608, 1899.11 .10 ; 611, 1899.III.3 ; 613, 1899.III.17 ; $616,1899 . I V .7 ; 620,1899 . V .5 ; 624,1899 . V I .2 ; 631,1899 . V I I .21 ; 636$, 1899.VIII.25; 644, 1899.X.20. Lehen emanaldia (Quelques règles de la prosodie ou art poétique basque) Nouvelles du Pays izeneko sailean argitara zen ; ondorengoak, Art Poétique Basque izenpean, sail beregain modura edo.

Geroztik ez bide da argitaratu. Eta aipatu kazeta eskuraerraza ez denez, areago, euskal literaturaren eskuliburuetan ere ia aipamenik ez denez, ezagutzeko aukerarik ere ez da izan. Hortaz, duela ehun urte argitaratu poetikalan hau berrargitaratzeari egoki iritzi diot, kontutan harturik, gainera, D'Abbadieren Lore Jokoen garaikoa dela. Ezen Zaldubyren xedea tradiziozko euskal neurkera zein den irakastea bada ere, argi dago Lore Jokoen garaiko neurkera dela hark tradiziozkotzat hartzen eta erakusten diguna.

Zaldubyren neurtiztiaren bigarren ekoizpen honetan kantuen testuetan lehen argitalpenean (Eskualduna kazetan) ageri diren hutsak zuzendurik agertarazten ditugu hemen, ustez behintzat. Leloren Kantukoak dauden-daudenean utzi ditugu, ordea. Bestalde, lehen edizio horretan latinezko kantuetako hitz batzuetan ae eta oe berdin idatzita baitatoz, bata edo bestea hautatu behar izan dugu. Hortaz, forma zuzendua markatu dugu, asterisko bidez, eta ondoren Eskualduna kazetan agertu forma jarri, beste asterisko baten ondoren.

(Eskualduna 608, 1899-II-10, Nouvelles du Pays)

L'Eskualduna du 20 janvier nous a donné, sous la signature de Paristarra, une fable ou apologue en chanson basque : Arraina eta bere umeak, qui est une des meilleures compositions basques de notre temps.

Voilà ce qui s'appelle composer selon toutes les règles de la vraie prosodie basque.

Nous voulons à cette occasion exposer ici même les plus élémentaires au moins de ces règles:

\section{Quelques règles de la prosodie ou art poétique basque}

1. Les Basques n'ont jamais fait de vers qu'en couplets réguliers destinés à être chantés ou même déclamés comme dans les pastorales. 
2. Le Basque ne reconnaît ni vers libres ni vers blancs. Il n'a égard qu'au nombre de syllabes, fussent-elles longues ou brèves. Les hiatus dans les mots qui se succèdent, même aux hémistiches, sont seulement tolérés.

3. Les rimes dans les vers basques ne vont pas seulement deux par deux : elles doivent être les mêmes dans toute la durée de la strophe même la plus longue. Quand il y a lieu de faire croiser deux rimes différentes ces deux mêmes rimes croisées, et pas d'autres, doivent régner dans tout le couplet.

4. Dans les vers qui se terminent par deux voyelles $e a, i a, o a, u a$; ea par exemple ne peut pas rimer avec $i a$ ni avec oa ni avec ua et réciproquement.

5. La même finale d'un verbe, non au participe, telle que naiz, dut, zen, dio, zion, duzu, etc., ne peut pas se répéter comme rime dans le même couplet.

6. Les Basques sont friands de la rime riche : la rime suffisante leur suffit à peine.

7. Deux couplets à la suite ne devraient jamais être de même rime et même de rime peu différente.

8. Les mesures des vers sont aussi diverses dans le basque que dans toute's les autres langues, elles dépendent de l'air ou musique adoptée par le poète.

Il y a des vers de quinze pieds avec hémistiche entre les $8 \mathrm{e}$ et $7 \mathrm{e}$ pied. Il est mieux d'en faire deux vers de 8 et de 7 de rime différente se croisant avec les rimes des vers pareils subséquents, comme dans l'hymne latin Pange lingua gloriosi...

Le vers basque le plus usité, l'alexandrin basque, est de treize pieds, avec hémistiche au $7^{\mathrm{c}}$. Les Basques d'Espagne l'appellent zortziko, - - huitaine - et l'écrivent en huit lignes, le couplet de vers de cette mesure, 7 et 6 , qui se compose ordinairement de quatre vers.

Il y a aussi des chants composés de véritables hexamètres semblables à l'hexamètre français.

Les vers de onze et dix pieds alternés ont l'hémistiche après le quatrième pied et ils doivent avoir deux rimes différentes croisées.

L'hemistiche dans le vers basque est soumis à des règles plus sévères que dans toute autre langue. Il équivaut à la fin d'un vers. Ainsi, entre les deux mots aita et ona, il ne peut y avoir d'hémistiche ni entre maite et zaitut.

9. Les vers au-dessous de dix pieds n'exigent pas la coupure fixe de l'hémistiche.

Nos poètes basques ou bardes de campagne n'ont pas besoin qu'on leur enseigne ces règles. Les plus illettrés d'entr'eux les observent naturellement, instinctivement. C'est à l'usage de certains compositeurs lettrés, habitués à la prosodie française ou espagnole, que nous publions ces notions particulières et spéciales à la prosodie euskarienne.

Dans un prochain numéro de l'Eskualduna nous signalerons comme éclaircissement divers morceaux de poésie basque, où tel ou tel numéro des susdites règles se trouvent violées.

Nous recommandons aux amateurs de conserver le présent article. 


\section{Art poétique basque}

\section{(Eskualduna 611, 1899-III-3)}

Dans un des derniers numéros de l'Eskualduna, nous avons publié une ébauche de notice sur la prosodie ou art poétique basque...

Ce travail nous était depuis longtemps réclamé par divers amateurs et aspirants versificateurs du pays, ainsi que par maints philologues bascophiles étrangers. On nous en a su gré plus que nous aurions osé nous y attendre. Mais aussi on nous engage à recommencer avec un peu plus de développement, ce qui nous semblait suffire comme simple ébauche. Est-ce un vrai traité sur la matière qui nous serait demandé ?... Nous ne savons si nous suffirons à la tâche...Essayons et recommençons :

\section{$I$}

Nous avons commencé ce travail en indiquant 9 règles principales en vigueur depuis des siècles dans la poésie populaire basque : règles dont, pour notre honneur national, il importe de maintenir mieux que jamais la tradition.

Il est bien à présumer que ces règles de la versification basque ne remontent guère au delà du XII ${ }^{\circ}$ siècle, et il est probable aussi que les chansons de geste de cette belle époque et particulièrement quelques hymnes rimés de l'Église leur servirent de modèles.

Les tercets monorimes du Dies irae, les quatrains de Jesus dulcis memoria, les stances du Lauda Sion, surtout les deux dernières, avec la même rime riche répétée quatre fois, durent infiniment plaire à l'oreille des Basques, et cette monorimie que dans ces temps reculés ils adoptèrent pour leurs chants régionaux, a été religieusement maintenue comme essentiellement populaire jusqu'aujourd'hui.

La poésie est une des choses qui tiennent le plus aux fibres intimes d'une nation. Chaque race a la sienne, chaque idiome surtout y accuse sa distinction. Le Basque trouve dans ses chants un des éléments de son patriotisme; pourquoi en modifierait-il les lois pour imiter l'étranger? N'a-t-il pas déjà par ailleurs assez fusionné avec les ravisseurs de son originalité, de ses droits et de ses libertés?

Depuis quelques années, cependant, il se manifeste une fầcheuse tendance vers un modernisme exotique. Des compositeurs, doués certes d'ailleurs d'un vrai souffle poétique, en prennent trop à l'aise avec la vraie et traditionnelle prosodie basque, et parfois se font couronner dans les concours : l'aveu et des protestations nous en sont venus même du côté de l'Espagne où pourtant Iparragirre fut un maître si correct.

Le contact, l'influence, l'habitude des littératures ambiantes ne devrait pas nous faire ainsi désapprendre les règles de notre art poétique euskarien; moins encore devrions-nous encourager certaines reproductions ou traductions serviles de romances attendries et trop connues des poètes qui ne sont ni de notre langue ni de notre sang.

Nos bardes ou bertsularis illettrés de campagne sont, sans qu'ils s'en doutent, malgré les incorrections qui échappent à leurs improvisations ou à leur manque de soin, les plus sûrs observateurs des règles de la versification basque : on dirait 
qu'ils y procèdent en vertu de quelque don atavique, sans aucune étude d'une pareille prosodie. Parmi eux, le meilleur modèle à citer, dans ces derniers temps, est le vieux Otchaldé, de Bidarray. C'est également sans étude, sans même avoir entendu jamais énoncer à d'autres, que chaque Basque, dès son jeune âge, pourvu surtout qu'il ne sache aucune autre langue que la sienne, conjugue si correctement son verbe si prodigieusement savant et si ingénieusement compliqué dans ses combinaisons.

Nous n'avons rien à apprendre aux poètes basques de nos villages : ils ne font que les fautes qu'ils sont impuissants ou trop négligents à éviter : que ne songentils à soigner et à limer leurs hâtives compositions?

Ils ont pourtant conscience de leurs fautes ; car dans les concours d'improvisation, par exemple, il n'est pas rare de les voir souligner eux-mêmes par leur quelque geste de dépit ou quelque grimace de mécontentement, alors même qu'ils savent combien le jury qui les juge et le public qu'ils amusent, leur sont indulgents.

Pour les compositions écrites avec préparation et loisir, toute indulgence envers les violateurs des règles serait absolument condamnable.

Entre une pièce médiocre de pensées, mais de facture correcte, et une autre de souffle plus poétique, mais irrégulière et fautive comme versification, la préférence devrait être donnée à la première. Les admirateurs de la seconde se borneraient à dire : "Que n'est-elle écrite en prose !"...Or, cette prose elle-même, s'imagine-t-on quels soins elle exige pour se produire dans toute la beauté de sa perfection? il en est qui l'estiment, au moins d'aussi savant et délicat maniement que la meilleure versification; car la phraséologie basque, sans rien changer aux lois qui depuis plusieurs milliers d'années régissent cet idiome, est susceptible d'un perfectionnement indéfini.

C'est particulièrement pour les compositeurs lettrés basques habitués à d'autres langues, aux dépens peut-être de ce qu'ils devraient savoir de la leur, que nous avons entrepris d'exposer les règles traditionnelles de l'art poétique euskarien.

\section{II}

$\mathrm{I}^{\text {ri }}$ règle. - La versification basque n'admet pas les vers libres se succédant sans mesure régulière ou sans rime.

$2^{e}$ règle. - Toute poésie basque en vers doit se composer de strophes ou couplets réguliers avec ou sans refrain, destinés à être chantés sur un air qui en règle les mesures et les cadences. Bien des Basques ne savent s'inspirer et composer qu'en chantant.

$3^{\mathrm{e}}$ règle. - Le vers basques est syllabique et non métrique, c'est-à-dire qu'il se règle non d'après la valeur des syllabes longues et brèves, mais selon leur nombre seulement. Les hiatus dans la rencontre des mots, même aux hémistiches, sont tolérés. Les élisions ne sont admises que le moins possible et seulement dans la rencontre de deux mêmes voyelles et dans quelques autres cas où elles font un heureux effet. 
$4^{e}$ règle. - Les rimes dans les vers basques ne vont pas seulement deux par deux ; elles doivent être de même son dans toute la durée du couplet, quelque multipliés que soient les vers de ce couplet.

$5^{e}$ règle. - Quand il y a lieu de faire croiser deux rimes différentes, ces deux mêmes rimes croisées doivent continuer jusqu'à la fin de la strophe. La richesse de la rime est très recherchée dans les vers basques, et souvent elle s'impose.

$6^{c}$ règle. - Deux voyelles ne s'élidant jamais en basque dans le même mot, deux et même trois voyelles successives constituent autant de syllabes distinctes. Dans les vers qui se terminent par deux voyelles, comme $e a$, ia, oa, ua, ea ne peut pas rimer avec $i a$, ni avec $o a$, ni avec $u a$, et vice versa.

$7^{*}$ règle. - Deux strophes à la suite ne doivent pas être de même rime ou aussi de rime peu différente.

$8^{\mathrm{e}}$ règle. - Dans les vers de dix syllabes et au-dessus, la césure ou hémistiche est de rigueur, elle doit marquer une suspension de sens soumise elle-même à certaines règles plus rigoureuses dans le basque que dans aucune autre langue. C'est comme si les deux parties du vers coupées par la césure constituaient deux vers distincts : en effet, les Basques d'Espagne écrivent d'ordinaire en deux lignes chaque vers comptant plus de dix pieds.

$9^{e}$ règle. - Les mesures des vers sont aussi variées en basque que dans les autres langues. Leurs nombres de pieds, leurs cadences, leurs hémistiches, le nombre de vers de chaque couplet sont déterminés par l'air sur lequel ils doivent être chantés.

(A suivre)

\section{Zalduby.}

(Eskualduna 613, 1899-111-17)

(Suite)

\section{III}

La versification la plus usitée, la plus classique, la plus commune aux sept provinces basques est le sept-six de 13 pieds, coupé par l'hémistiche entre le septième et le sixième pied. Son similaire en français est, pour le mouvement rythmique, tout ce qui se chante sur l'air du cantique si connu :

Je mets ma confiance

Vierge, en votre secours ;

Servez-moi de défense,

Prenez soin de mes jours ;

Et quand ma dernière heure

Viendra fixer mon sort,

Obtenez que je meure

De la plus sainte mort.

Il y a ici huit vers à rimes croisées, au lieu que dans le basque il n'y en aurait que quatre, les quatre de 13 pieds, mais les quatre rigoureusement de même rime. 
Les Basques d'Espagne les écrivent en huit lignes, comme s'il y avait huit vers :

Gernikako arbola

Da bedeinkatuba,

Euskaldunen artean

Guztiz * maitatuba : * Gutiz

Emanta zabaltzazu

Munduan frutuba :

Adoratzen zaitugu

Arbola santuba.

Voilà ce que les Guipuscoans, très épris des originalités de leur dialecte plus coutumier pourtant de licences grammaticales que notre labourdin, observent toujours rigoureusement, en l'appelant leur zortziko, ce qui veut dire à huit vers ou huitain en français.

Dans les strophes de cette mesure-là, la même rime répétée huit fois serait un luxe de fantaisie qui n'offre guère d'exemple. Cela n'aurait rien d'irrégulier. Mais au moins les quatre vers de 13 pieds doivent être rigoureusement monorimes.

Dans mes débuts de vingt ans, il y a un peu plus de demi-siècle, en collaborant avec Elissamburu, je trouvai qu'il était plus facile de composer en changeant de rime à tous les deux vers à l'imitation de la versification française (sauf que celle-ci est assujettie à l'alternance des rimes masculines et féminines); et c'est alors que je fis la première ébauche d'une chanson très défectueuse qui depuis s'est répandue dans le public par des copies de plus en plus fautives:

Ene hauzotegian

bi senhar emazte

Bakean bizi dire

hasarretu arte.

Guardia elgarrekin

behin makhurtzetik.

Heien chuchentzaileak

segur lanak ditik.

Au moins aurait-il fallu donner aux deux derniers vers une finale rimant avec les premiers ; par exemple :

Gerlan elgar menturaz

maiteago dute :

Hoien artean sarthuz,

nik pampak nituzke.

Malgré la vogue acquise par cette mauvaise composition, je n'hésitai pas à la remanier dans la suite, pour la rendre irréprochable; mais, chose singulière ! le public s'en tient encore à mon premier jet.

Les deux cantiques : Oi mirakulu guziz espantagarria...et Kristau gazte maitea, zuretzat naiz mintzo, sont également fautifs contre la règle $4 \mathrm{e}$; mais l'habitude de les chanter tels quels leur a donné comme une consécration, et nous n'oserions les corriger. 
Il y a lieu d'être plus sévère pour certaines poésies de même irrégularité, qui ont été couronnées dans nos concours de fêtes basques...

Nous n'en citons qu'une aujourd'hui :

Bakharrik bizitzeaz unathua franko

Chede zerbait banuen lagun bat hartzeko;

Bainan orai andreak zombat ez du behar

Ez ez nahiago dut egon muthil zahar.

Ce changement de rime dans le même couplet de ce zortziko déchire vraiment l'oreille basque. Deux rimes différentes, jamais davantage, peuvent se produire, mais toujours croisées ou alternées quand les vers ont moins de syllabes, comme nous l'expliquerons plus bas.

Il y a des poésies basques très correctes, qui n'ont que deux vers à chaque couplet ; par exemple :

Iruten ari nuzu khilua gerrian,

Ardura dudalarik, nigarra begian.

ou encore :

Gobernamenduetan zoin da zuzenena?

Ethorkiz legez eta obraz hala dena.

Refrain $\mathrm{Ai}$ ai ai ! mutilla, obraz hala dena.

Les quatre hémistiches de ces deux vers.

Parfois, le poète se donne le luxe de faire rimer chacun des hémistiches de ces deux vers; ou bien, par le même son syllabique (ce qui est rare dans cette mesure de 7-6), ou bien par deux rimes différentes croisées :

Gure lehen aitamen

Bekhatuak ez du

Sekulan Mariaren

Arima gibeldu.

(A suivre)

\section{Zalduby}

(Eskualduna 616, 1899-IV-7)

(Suite)

La versification en treize syllabes, avec hémistiche à la septième, et en couplets monorimes, est ce qu'il y a de plus basque, de plus usité et de plus régulièrement* observé parmi les Basques. (*regulièrement)

Celle de douze syllabes est assujettie à des règles identiques, ou au moins devant l'être, bien qu'elle ressemble à l'alexandrin français à rime masculine. Les poètes basques en font rarement usage. Le cantique des mystères du Rosaire :

Adoratzen zaitut Berbo dibinoa,... 
en serait un modèle irréprochable si ses couplets étaient monorimes, condition à laquelle a cru devoir bien se soumettre l'auteur du cantique du Saint Sacrement :

Jesus, amodioz - bihotza gaindian,

Gurekin osoki - bat egin nahian ;

Gure hazkurri zen - azken afarian,

Guk jateko egin - bere haragian.

$$
\text { IV }
$$

Il y a encore des versifications plus faciles, qui ont $15,16,17$ et jusqu'à 18 syllabes à chaque vers, avec hémistiche dépendant de la cadence musicale qui en règle la place. Ordinairement cet hémistiche coupe le vers, en 8 et 7 dans les 15 syllabes; en 8 et 8 dans les 16 syllabes; en 9 et 8 dans les 17 syllabes, et en 9 et 9 dans les 18 syllabes. Au delà de 18 syllabes deux vers distincts s'imposent, ou de même rime ou de deux rimes différentes; mais aussi les mêmes toutes les deux, dans toute la durée du couplet, quelque long qu'il soit.

Voici quelques exemples de la versification dépassant les 13 syllabes à chaque vers. Nous marquons l'hémistiche par un trait.

$I^{\circ}$ Vers de 15 pieds.

Biziaren primaderan - harmetara joaitea,

Zorigaitzik dorpheena - zitzautan jasaitea.

Desertatuz uste nuen - hobeki izaitea

Phentsamendu zoro horrek - egin zautan kaltea.

Il est certain qu'il y a plus de mérite à faire deux vers distincts de 8 et 7 pieds avec ces 15 syllabes d'un seul vers. Par exemple :

Laborantza gauza handi

Eta botheretsua ;

Hartan da lanean ari

Jainkoaren eskua,

Gizonaren eskuari

Berma dadin lothua.

Mais les vers de cette mesure-là ne se font guère que pour des couplets à six vers. Saint Thomas en fit de semblables pour son hymne :

Pange lingua gloriosi

Corporis mysterium

Sanguinisque pretiosi

Quem* in mundi pretium *Quim

Fructus ventris generosi

Rex effudit gentium.

Voici la première strophe d'un cantique où les vers des mêmes mesures 8 et 7 , nous offrent, au lieu de deux seules rimes régulièrement alternées, un entrecroisement irrégulier de trois rimes:

Jaunak gauzka mundu huntan, Hemendikan zerurat : 
Bere ganat hartzekotan

Dohatsuen baltsarat ;

Altchatzagun gure bozak,

Kanta errepiketan,

Bozekin junta bihotzak

Zerurako hotsetan.

Toutefois, il faut convenir que cette rime en tan, ainsi quatre fois répété, ne manque pas de produire à l'oreille quelque heureux effet; mais la même combinaison ne subsiste pas dans les couplets suivants. Remarquons le troisième, qui est vraiment beau comme pensée :

Gure ilhumpe lodiak

Dire hor suntsituko:

Zuk agertu bekhokiak

Gaitu hor argituko.

Argitu suerte guziak,

Egia guziekin,

Ditezke gure argiak,

$\mathrm{Zu}$ ikhustearekin.

Ici, les quatre rimes en iak sont bien à leurs places et rangs réguliers; mais la consonance en $k o$, en se croisant avec iak, aurait dû se produire aussi quatre fois. Les deux couplets seraient dans les règles, modifiés par exemple comme il suit :

Cristauak gure izantzak

Ez dire mundu huntan;

Zerurako esperantzak

Gerauzka lorietan ;

Altchatzagun gure bozak.

Kanta errepiketan ;

Bozekin junta bihotzak

Zerurako hotsetan,
Gure ilhunpe lodiak

Han dire suntsituko ;

Jainkoaren arphegiak

Gaitu han argituko,

Han egiaren argiak

Gaitu inguratuko,

Han dohatsuen loriak

Iraunen du bethiko.

\section{$2^{\circ}$ Vers de 16 et de 17 pieds.}

Ici, nous présentons comme exemple, sinon comme modèle, la poésie couronnée au concours de 1898 :

\section{Irrintzina bat mendian}

Gau eder ba - tek ninduen - hatzeman mendi gainetan ;

Iguzkia - aintzinean - emeki etzan zitzautan :

Azken abereak etchera - zohazin ene oinetan,

Bazterrak inharosiz beren - joare soinu ederretan.

L'air ou la musique appliquée à cette chanson semblerait exiger deux repos ou césures à chacun des deux premiers vers. Nous regrettons de ne pouvoir la noter ici pour ceux qui ne la connaissent pas.

Est-ce une fois que la nuit eût surpris le berger, que le soleil se coucha doucement devant lui ?...est-ce que les clochettes de ses brebis faisaient trembler 
autant qut cela les échos de la montagne ?...ce n'est pas le lieu ici de critiquer ce côté de la composition.

Lehen iza - rrak doidoia - ziren orduan agertzen

Harrabots gu — ziak ere - hasiak ziren eztitzen

Gaua, hura ere ari zen — kapa beltzaren hedatzen

Bitartean etche batzu... - oraino ikus zitezken.

Il manque un pied à ce dernier vers. Il aurait fallu :

Bizkitartean etche batzu oraino ikus zitezken

Hor sumatzen - dut artzain bat - - mendi artetarik kantuz

Zakizkan Es - kualdun aire - zaharrak charamelatuz

Boz hura bakharrik orduan - mendi gain hetan adituz

Beha nindagon lorietan - eta burua zutituz.

(Errac bada : burua goitituz.) Le terme zutituz s'emploie pour le reste du corps, jamais pour la tête seule.

Dans ce couplet, le vers

Zakizkan Eskualdun aire — zaharrak charamelatuz,

il y a une faute grave contre les règles basques de l'hémistiche. Ces deux mots aire et zaharrak ne peuvent ici être séparés pour marquer un repos, pas plus que dans ce vers de Racine:

Ma foi, j'étais un franc - portier de comédie.

les deux mots franc et portier n'auraient dû se disjoindre en césure.

Et cependant le poète de l'Irrintzina aurait pu être très correct en disant :

Eskualdunen - aire chahar - guziak charamelatuz.

Les règles de la césure dans le vers basque ont de ces délicatesses que nos bardes de campagne savent très naturellement observer. Peut-être, au cours de cette étude, parviendrons-nous à donner une formule précise à ces règles de l'hémistiche.

C'est surtout de la poésie basque qu'il est vrai de dire que les paroles sont esclaves de l'air sur lequel elles sont composées et chantées: on le voit bien à la façon dont la musique impose ses coupures et les affirme dans les hémistiches.

Nous étonnerons peut-être bien des gens en disant que la race euskarienne a sa musique propre, ses airs à elle, ses mélodies d'une antiquité au moins de tradition qui semblerait devoir les ériger en lois. Les poètes basques ne devraient jamais s'en écarter, moins encore les dédaigner, en adoptant une musique exotique surtout française. Les Basques n'ont rien à gagner à tout ce que les meilleurs et les plus vrais d'entre eux appellent frantchimankéries. Dans toute manifestation libre de sa vie, le Basque digne de ce nom, se doit d'affirmer son instinct d'autonomie. Dans le chant d'Irrintzina bat mendian, sur l'air de Sor lekhua utziz geroz...de notre grand poète Elissamburu, la mélodie de vrai caractère basque fait absolument défaut : ce qui surprend de la part de l'illustre euskarien qui fit choix de pareille musique. 
A la fin de cette étude, nous aurons un chapitre spécial, où nous nous inspirerons des appréciations de haute autorité d'un des plus grands maittres de notre siècle sur la mélodie basque. (A suivre.).

\section{Zalduby.}

\section{(Eskualduna 620, 1899-V-5)}

(Suite)

Voir les numéros 611, 613,616 de l'Eskualduna.

Le vers basque étant simplement et absolument syllabique, sans différence de syllabes longues ou brèves, autres que celles que les fait telles le rythme musical ; ... sans distinction non plus de rimes masculines ou féminines...(Le basque n'a de féminin que le verbe tutoyé parlant à la femme)...Dans ces conditions, où le jeu de la versification est bien simplifié, il n'est pas malaisé de composer correctement des vers de 17 et de 18 pieds, comme on en couronna, dans un des premiers concours d'Urrugne, il y a une quarantaine d'années. Il est vrai qu'en latin aussi il y a des hexamètres de 17 pieds, sans compter les syllabes élidées. En voici un de Virgile :

Dulcis et alta quies placidaeque simillima morti.

Un autre encore de Prudence (de son vrai nom Galindo), basque d'origine d'Huesca de la Vasconie espagnole :

Praemia saltatrix poscit funebria virgo Joannis caput.

Mais la savante métrique observée dans ces vers à dactyles et spondées, acquiert à leurs dix-sept syllabes composant six pieds, le mérite d'autant de difficultés vaincues.

Des vers de plus de dix-huit pieds seraient évidemment indignes d'un poète basque tant soit peu exercé : on ne les tolère, dans une certaine mesure, que dans les improvisations, les boutades hâtives, et les toasts chantés des joyeux banquets.

Les vers de seize et de dix-huit syllabes d'un chant tel que l'Irrintzina, fait à tête reposée, ont donc atteint l'extrême limite de ce qui est permis. Sur la mélodie qui règle le rythme de cette pièce, pour chaque vers basque, le français en ferait deux.

Volontiers nous donnerions raison à ceux qui exigeraient ces deux vers, au delà de quinze pieds, si une tradition très tolérante n'en avait déjà admis jusqu'à dix-huit. Mais aussi, dans ces larges limites permises, faut-il que le poète, surtout celui qui se produit dans un concours, ne présente que des vers d'une facture et de locutions irréprochables, ce qui n'est pas certes le cas du dernier chant couronné.

Irrintzina bat mendian...le choix seul de ce sujet fait honneur à l'auteur de la pièce. Voilà qui est digne d'inspirer un poète basque, comme il avait déjà inspiré le prestigieux prosateur français, M. Loti, dans son Ramuntcho.

Comme hors-d'œuvre dans le cours de cette étude, nous ne pouvons résister au plaisir de citer ici même la page de l'académicien admirateur des Basques: 
"Mais tout à coup, de cette barque qui était si tranquille et qui n'avait plus que l'importance d'une ombre à peine réelle au milieu de tant de nuit, un cri s'élève, suraigu, terrifiant; il remplit le vide et s'en va déchirer les lointains...Il est parti de ces notes très hautes qui n'appartiennent d'ordinaire qu'aux femmes, mais avec quelque chose de rauque et de puissant qui indique plutôt le mâle sauvage ; il a le mordant de la voix des chacals et il garde quand même on ne sait quoi d'humain qui fait davantage frémir ; on attend avec une sorte d'angoisse qu'il finisse, et il est long, long, il oppresse par son inexplicable longueur... Il avait commencé comme un haut bramement d'agonie, et voici qu'il s'achève et s'éteint en une sorte de rire, sinistrement burlesque, comme le rire des fous...

"Cependant, autour de l'homme qui vient de crier ainsi à l'avant de la barque, aucun des autres ne s'étonne ni ne bouge. Et après quelques secondes d'apaisement silencieux, un nouveau cri semblable part de l'arrière, répondant au premier et passant par les mêmes phases, - qui sont de tradition infiniment ancienne.

"Et c'est simplement l'Irrintzina, le grand cri basque, qui s'est transmis avec fidélité du fond de l'abîme des âges jusqu'aux hommes de nos jours, et qui constitue l'une des étrangetés de cette race aux origines enveloppées de mystère. Cela ressemble au cri d'appel de certaines tribus Peaux-Rouges dans les forêts des Amériques ; la nuit, cela donne la notion et l'insondable effroi des temps primitifs, quand, au milieu des solitudes du vieux monde, hurlaient des hommes au gosier de singe.

“On pousse ce cri pendant les fêtes, ou bien pour s'appeler le soir dans la montagne, et surtout pour célébrer quelque joie, quelque aubaine imprévue, une chasse miraculeuse ou un coup de filet heureux dans l'eau des rivières."

Oh ! que tout cela est ingénieusement imaginé, et merveilleusement décrit en prose française! mais aussi combien cela méritait d'être reproduit dans notre bel idiome euskarien!

Le poète basque de l'Irrintzina a été très heureux dans les détails vraiment poétiques dont il a encadré la reproduction de cette perle trouvée dans Ramuntcho: que ne les a-t-il aussi correctement dits que poétiquement pensés?

Pour répondre de suite à certains désirs exprimés, et édifier ceux qui n'ont guère jamais regardé de près à la correction et au soin exigés par la bonne versification basque : ... afin de faciliter, aussi d'avance, d'autres notions que nous avons à exposer plus loin, nous allons, avant de clore ce chapitre des vers à longueur exagérée, relever les fautes les plus apparentes du chant de la dernière actualité, - l'Irrintzina, — à la suite de celles qui ont été déjà signalées précédemment :

\section{$4^{\mathrm{e}}$ couplet}

Bat batetan, kantu orde, oihu latz bat dut aditzen

Bere samintasunean 1 naualarik harritzen 2.

Mendi mazeletan* harri ko 3 - tor zaharrak zituen jotzen 4 *mazelelan

Eta lekhu basa hetako oihartzunak iratzartzen.

1 Samintasunean, après latz - un cri rude dont l'aigreur m'effraie - fallaitil bien ces deux épithètes? 2 A ce second hémistiche il manque un pied; et puis voilà un Basque qu'un irrintzina effraie. Il eût mieux valu qu'il dît : "Bainan 
Eskualduna bainaiz ez nau ni hambat izitzen." 3 Ici césure impossible. Harri kotor est un terme absolument impropre. 4 Voilà un second hémistiche qui, chanté, serait "tor zaharrak* zituen" hélas! il n'y a pas de place pour le mot jotzen qui est pourtant la rime. (*zahalrak). "Mendi mazeia hek guziak urrundik zituen jotzen" eût été plus correct.

$$
5^{e} \text { couplet }
$$

Oihu gora, garratza 5 zen nola emaztearena,

Azkarra eta bortitza nola gizonkiarena ;

Artetarik iduri zuen basa ihizi batena;

Zertan edo hartan hargatik, oraino jendearena.

5 Garratza ici est bien défectueux, c'est samina qui s'imposait surtout appliqué à emaztearena. Ce 5 e couplet ainsi que le 6e sont les meilleurs de la pièce.

$$
6^{\mathrm{e}} \text { couplet }
$$

Luzez zuen iharrosi mendi hetako bortua, 6

Iduri zuen hastean norbeit zela minhartua, Irri lotsagarri batean zen aldiz akhabatua, Bide chedran nindagorik ni oraino baratua.

Mendi et bortua. Bien des Basques trouveraient là un pléonasme. Nous voulons croire que par mendi hetako bortua l'auteur a voulu dire le désert ou la solitude de ces montagnes.

$$
7^{\circ} \text { couplet }
$$

Ibañetan ere lehen irrintzina aditu zen,

Arrolani zitzaiola odol guzia gatzatzen : 7

Alferretan ditu mendiak deihadaraz 8 dardaratzen;

Eskualdunen irrintzineak $9 \mathrm{du}$ ichiltzera bortchatzen.

7 Il est inadmissible que le sang de Roland fût capable de se figer de frayeur comme le sel. 8 Deihadarra, dans le basque actuel, veut dire cri, hurlement de douleur et d'appel au secours, et ne s'emploie qu'au pluriel : deihadarrez dago. Le poète de l'Irrintzina a-t-il voulu donner le nom de deihadarra (au singulier) exprès et à dessein, à l'oliphant de Roland...dei - adarra, appel - corne ?...ce serait merveilleusement appliqué selon la vraie et évidente étymologie de ces deux mots dei et adarra, dont probablement les Basques, dans le cours des siècles, auraient changé la signification littérale. M. Barbier aurait dû accompagner son terme d'une parenthèse explicative (dei — adarra - corne d'appel), rien que cela. Ce mot deihadarra, ainsi entendu, fait penser aux sonneries d'alarme primitivement employées par les Basques dans leurs montagnes. Ces sonneries de corne ont lieu encore en certaines localités, notamment à St-Just, pour l'appel des troupeaux ; elles retentissent aussi sous le nom de tûtak et turrutak, dans tous les charivaris basques. 9 Irrintzineak, vrai barbarisme-cheville : il était bien simple d'avoir les neuf syllabes requises par ce premier hémistiche en disant : Eskualdunaren irrintzinak, ou bien Eskualdunen irrintzinak $d u$ - lasterchko ichilarazten. (A suivre)

\section{Zalduby.}


(Eskualduna 624, 1899-VI-2)

(Suite)

(Voir les numéros $611,613,616,621$ de l'Eskualduna).

Dans notre dernière leçon, nous avons relevé, dans le chant au souffle très poétique de l'Irrintzina, des fautes contre la versification basque, dont une bonne partie serait, paraît-il, imputable soit au compositeur de l'imprimerie, soit à quelque correcteur de la commission du concours. Nous nous en voulons de n'avoir pas connu à temps l'édition génuine que de son côté er! avait tiré l'auteur.

M. de Jaurgain, notre éminent fouilleur d'archives basques, a eu la gracieuseté de nous faire ces jours derniers l'inappréciable hommage de sa brochure si savamment documentée, sur quelques légendes poétiques du pays de Soule, où il nous fait l'historique et nous donne les dates de plusieurs vieilles chansons qui ont encore de la vogue dans tout le Pays Basque français. Nous extrayons d'abord le premier couplet de la fameuse ballade de Berteretche, qui fut composée entre 1434 et 1449.

$\begin{array}{lllllll}1 & 2 & 3 & 4 & 5 & 6 & 7\end{array}$

Haltzak eztu bihotzik,

1 2345678

Ez gaztamberak ezurrik :

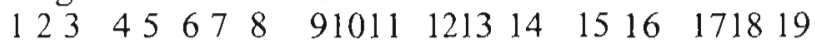

Enian uste erraiten ziela ai tu nen semek gezurrik.

Le $11^{\mathrm{e}}$ pied du $3^{\mathrm{e}}$ vers est là de trop selon la rigueur de la règle : à l'entendre chanter, cette faute passe inaperçue. Rien que par ce délicieux spécimen, on voit que depuis quatre siècles la langue basque n'a pas changé d'un iota.

Atharratz jauregian bi zitron doratu...ou bien la version primitive, d'après $\mathrm{M}$. de Jaurgain : Ozaze jaurgainian bi zitron doratu, Atharratzeko joanak (sic) bata du galthatu...et le reste, se chantait déjà vers 1631, époque de la mort de Marie de Jaurgain, l'héroïne de la chanson, pour lors sexagénaire. Cette inoubliable poésie étant en alexandrins basques classiques et ordinaires de 7-6 ou 13 pieds; dans ce chapitre de la versification à plus de 15 syllabes, nous ne faisons que la mentionner pour en signaler la haute vieillesse.

En voici une autre de la même époque (1664) en pleins dix-huitains, dont le premier couplet est vraiment suggestif :

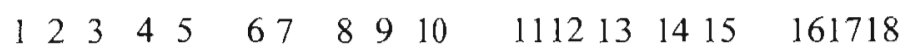

Goizian goizik jeiki ninduzun, ezkuntu nintzan goizian;

Bai eta ere zetaz beztitu, ekhia jelki zenian.

Etchek' andere zabal ninduzun eguerdi erditan

Bai eta ere alharguntsa gazte ekhia sarthu zenian.

Dans l'origine, le troisième vers devait être plus correct et complet ; comme ceci, par exemple :

Etchek'andere zabal ninduzun ber egunaren erdian,

et le quatrième aussi serait mieux comme il suit :

Bai eta gazterik alhargundu ekhia sarthu zenian. 
Nous aurons à signaler encore dans la suite la défectuosité des rimes de mêmes finales de verbes, telles que zenian et autres, répétées dans le même couplet.

Admirateurs de poésies aussi anciennes et aussi savoureusement basques que celles que nous venons de citer, nous les aimons jusques dans leurs imperfections prosodiques, que nous croirions ridicule de corriger autrement que comme leçon de faute à éviter dans les compositions modernes faites à tête reposée.

V

Vers de onze et dix syllabes

Les vers basques de onze syllabes continuées dans cette même mesure tout le long d'un couplet sont jusqu'ici extrêmement rares. Nous n'en avons sous la main qu'un exemple en cantique d'avant le sermon : il est très ancien. En voici la première strophe, que nous produisons, comme du reste tous autres exemples, en orthographe moderne :

Heda, Jauna, heda zure argiak,

Zuk argituz har detzagun egiak.

Gizon lagun igortzen dauzkutzunak,

Argi nola gaitzake gu gizonak.

L'hémistiche est bien là à sa place ; car à moins d'exception imposée par un rythme particulier de la mélodie, dans tout vers, soit de onze soit de dix syllabes, l'hémistiche est de règle rigoureuse entre la quatrième et la cinquième : c'est ce qui avait évidemment embarrassé l'auteur de ce cantique dans le quatrième vers.

Ce n'est pas tout. Il aurait aussi fallu que les quatre vers eussent la même rime, ou bien, à la rigueur, que les deux rimes différentes fussent croisées ou alternées, l'une terminant le ler et le $3 \mathrm{e}$ vers, l'autre le $2 \mathrm{e}$ et le $4 \mathrm{e}$. Il n'eût pas été malaisé d'arranger ce couplet de la façon qui suit :

Ai! igutzu, Jauna zure argiak;

Hunki zatzu, gure bihotz nagiak;

Entzutera goazin predikariak

Zure hitzez susta gaitzan guziak.

Ou bien encore, avec rimes croisées :

Ai! igutzu, Jauna, zure argiak,

Zure hitza ongi entzun dezagun;

Erratera dohazkigun egiak

Bihotzean barna sar dakizkigun.

Une versification plus usitée est celle qui fait croiser les vers de onze syllabes avec ceux de dix, soit en couplets monorimes soit en couplets à deux seules rimes différentes alternées.

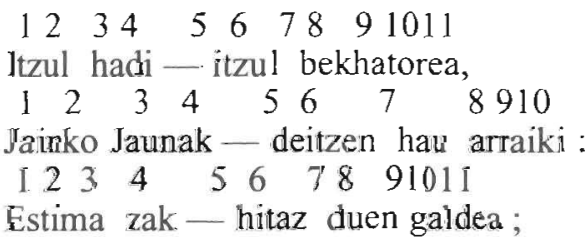


$1234 \quad 567 \quad 8910$

Eta emok - bihotza osoki.

C'est semblable au français :

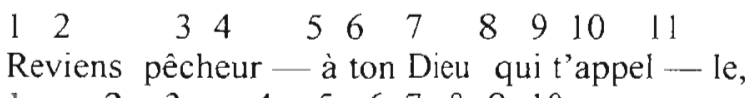

$\begin{array}{lllllllllll}1 & 2 & 3 & 4 & 5 & 6 & 7 & 8 & 9 & 10\end{array}$

Viens au plus tôt -. te ranger à sa loi :

$\begin{array}{lllllllllll}1 & 2 & 3 & 4 & 5 & 6 & 7 & 8 & 9 & 10 & 11\end{array}$

Tu n'as été - trop long-temps qu'un rebel-le,

$\begin{array}{llllllllll}1 & 2 & 3 & 4 & 5 & 6 & 7 & 8 & 9 & 10\end{array}$

Reviens à lui - puisqu'il vient jusqu'à toi.

Pour fortifier la comparaison, nous avons à dessein compté la finale muette du vers français comme syllabe accentuée.

Chose singulière, ce genre de vers, pourtant de très facile composition, n'a jamais encore, que nous sachions, été employé pour aucun chant profane, dans aucun Pays Basque, soit de France soit d'Espagne. Mais en revanche, now cantiques, et des meilleurs, nous en offrent de nombreux modèles. Ainsi, outre Itzul hadi... dont nous venons de donner le premier couplet, plusieurs autres tout aussi populaires, dont nous ne citons ici que le premier vers :

Mundu zoro tromperiaz bethea, etc.

Aditzen da trompeta lazgarria...

Huna Jauna ardi bat galtzen zena...

Othe da deus nesesario denik...

O arima Jainkoaz onetsia...

(A suivre.)

\section{Zalduby.}

(Eskualduna 631 1899-VII-21)

(Suite)

Voir les numéros $611,613,616,621,624$ de l'Eskualduna.

Les vers français de dix syllabes avec leur césure ordinaire après le $4 \mathrm{e}$ pied, n'ont, avons-nous dit déjà, leurs similaires en basque que dans les chants d'Église composés selon le même rythme français, la onzième syllabe muette de chaque vers féminin y étant pourtant reproduite comme accentuée et masculine. En réalité, même en français, la notation de la musique accuse bien aussi cette onzième syllabe.

Dans les chants profanes d'allure vraiment basque, les vers de dix pieds sont inusités jusqu'à présent, en tant que couplets rimés. Mais en revanche, les vers de dix-huit syllabes, équivalant chacun à deux vers de dix et huit pieds avec césure après le cinquième et rime au second vers seulement, sont d'un usage fréquent surtout dans les romances modernes du Guipuscoa.

Ume eder bat — ikusi nuen 10 


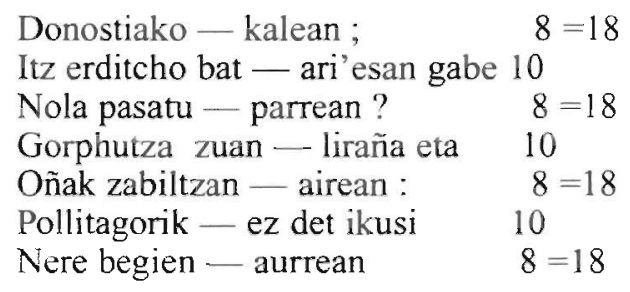

Dans le couplet suivant, la césure n'est requise qu'au ler vers :

Andre Madalen, - - Andre Madalen, 10

Laurden erdi bat olio $\quad 8=18$

Aitak jornalak - artzen badittu $\quad 10$

Berak pagaturen dio $\quad 8=18$

Il est vraiment commode de composer de la sorte quand la rime n'est qu'au 18e pied ; mais aussi a-t-on lieu d'être sévèrement exigeant à l'égard de toutes les autres conditions d'une composition correcte.

\section{VI}

Avec ces vers de 18 et 8 syllabes, nous entrons dans la catégorie des mesures, quantités, rythmes, césures les plus variés, auxquels nous ne pouvons assigner d'autres règles que celles imposées par les plus capricieuses mélodies. Voici la lre strophe d'un chef-d'œuvre :

Herriko besta - bigarrenean, 10

Berek dakiten - chokho batean ; 10

Lau andre, - bat alharguna ; - hiru mutchurdin - jarriak itzalean $20 *{ }^{*} 10$

Harri chabal bat belhaunen gainean 11

Ari ziren, ari ziren trukean. $\quad 10$

Remarquez ces différentes césures qui sont de rigueur aux trois premiers vers, et qui sont maintenues telles dans le cours de la chanson. Remarquez aussi ce troisième vers long de 20 pieds avec ses trois césures.

La même particularité se voit dans une autre pièce, la plus belle sans contredit et qui nous vient du même auteur :

$\begin{array}{ll}\text { Ikusten duzu - goizean, } & 8 \\ \text { Argia hasten - denean, } & 8 \\ \text { Menditto baten - gainean, } & 8 \\ \text { Etche ttikitto, - aintzin churi bat, } & 10 \\ \text { Lau haitz handiren - artean ; } & 8=18 \\ \text { Ithurriño bat - aldean, } & 8 \\ \text { Chakur churi bat - athean } & 8 \\ \text { Han bizi naiz ni - bakean. } & 8\end{array}$

Encore ici la césure est après la $5^{e}$ syllabe de chaque vers, même dans le demivers de 10 syllabes. Et ce demi-vers, pourquoi n'est-il pas un vers complet avec rime comme les sept autres du couplet? Pourquoi ces 18 pieds avec une seule rime s'allongeant ainsi au beau milieu du couplet ?...Est-ce une licence ? Notre éminent poète Elissambure pouvait sans doute se la permettre, et peut-être même 
la faire admettre comme règle dans ce cas particulier. Une vieille chanson des guerres carlistes, apprise dans notre enfance, lui en avait donné l'exemple. Il faut. entendre chanter cette ravissante poésie pour remarquer combien elle paraît régulière dans toute sa facture, et il est bien à regretter que tant de copies fautives, surtout l'édition de l'Eskualdun kantaria, qui s'est récemment répandue dans le public, en aient détérioré le texte primitif.

En dehors de ces singularités que nous venons de signaler dans la trame ordinairement si régulière de la versification basque, de l'ensemble de tous les modèles que nous tenons de la tradition purement basque et vraiment populaire, émerge la loi d'une seule et même rime riche, imposée à tous les vers, quelque nombreux qu'ils soient, dans la même strophe.

Sans doute nos bardes, et des meilleurs, ne composant guère jamais à tête reposée et dans le loisir d'un cabinet, ont maintes fois violé cette loi ; mais on voit que c'est toujours par impuissance ou défaut de soin : dès qu'ils le peuvent ils rentrent dans la règle, et même dans leurs infractions ils tâchent presque toujours d'employer une rime d'une assonance aussi rapprochante que possible de la rime qui leur fait défaut.

Le jury du concours Abbadien de 1898 accorda une mention honorable à une pièce guipuzcoane intitulée Nekhazari* dohatsua, dont le prologue en deux strophes de 7-6 pèche délibérément contre la règle des couplets monorimes* *Nekhabari; *monorismes.

Nere pipa chit bete, ta suaz pizturik

Keia goruntz, chistua lurrera botarik ;

Banetorren echeruntz bertsoak kantatzen,

Buruan berez berez ziranak sortutzen ;

Zeiñtzuek diran letraz gaur jarritakoak,

Izan arren balio oso guchikoak,

Ala ere nai nuke emenchen lenbizi,

Orain irakurtzera jendeari asi.

Et cependant, il est soigneux à l'observer dans les onze stances à cinq vers dont est composé son chant; en voici la première :

Fumatzen duela ere Erregek badakit nik,

Eizean aspertutzean asnas artzen jarririk ;

Ordean, artu ez arren onen belar merkerik,

Apostu egingo nuke ez diola gustorik,

Abanoari ark artzen, nik pipari lakorik.

Il reconnaît donc la justesse de la règle.

(A suivre). 
(Eskualduna 636, 1899-VIII-25)

(Suite)

Voir les numéros 611 , 613, 616, 621, 624, 631 de l'Eskualduna.

Voici des exemples de strophes à vers inégaux assujetties à des airs ou mélodies populaires particuliers au Pays Basque :

$\begin{array}{ll}\text { Hau da Ikhasketako } & 7 \\ \text { Mandoaren traza: } & 5 \\ \text { Lephoa mehe du'ta } & 7 \\ \text { Ilea latza, } & 5 \\ \text { Itchura gaitza : } & 5 \\ \text { Bastape guzitikan } & 7 \\ \text { Zorne eta baltsa, } & 7 \\ \text { Eta zer saltsa! } & 5 \\ \text { Kristaurik ez daiteke (bis) } & 7 \\ \text { Aldetik pasa. (bi) } & 5\end{array}$

Qui de nous ne s'est esclaffé de rire en chantant ou en entendant les vingt couplets de cette poésie burlesque due à un charbonnier, vrai Rabelais basque d’il y a déjà un siècle.

Sur cette même mesure, mais à rimes doublées, une satire contre les mauvaises langues fut présentée au concours de 1857 :

Gure herri chumetan

Zembat hitzuntzi

Zinez edo jostetan

Oro erasia.

Eta nahasi :...

Beha daude nor zertan

Hutsean kausi,

Nori pitz hauzi :

Deus ez nahiz bertzetan

Onik ikusi.

(bis).

Nos grands-pères aimaient aussi à chanter cette délicieuse goguenarderie du Bidarraitar :

Nork nahik zer nahi erranikan ere,

Bidarraitarra* nuzu, bai nahi ere, *Bidarraitara

Etcheko seme ona segurki halere.

Nahiz baden herrian hoberikan ere,

Baitut bortz haurride*,

*hauride

Enekin sei gire,

Oro adichkide :

Dothearen gainetik samurturen ez gire.

Ce premier couplet, depuis notre enfance, est dans toutes nos mémoires; il nous charme par son allure et son originalité, et nul jusqu'ici n'a songé à lui chercher noise pour sa répétition fautive du même mot ere employé trois fois comme 
rime, ainsi que de gire, qui l'est aussi deur fois. Les autres couplets de la même chanson sont plus réguliers.

C'est ici le lieu de signaler aussi deux de nos plus vieux et de nos meilleurs cantiques basques:

Itsasoan pareko

Mundu hau Salbamenduko

Chume handiak,

Garen guziak,

Etsaiarekin guduka,

Hertsaturik baikaguzka.

Gizona nun duk zuhurtzia,

Zer ez dakik

Ez dela deus ere bizia

Khe bat baizik

Higuin zak bihotzez mundua

Orai* danik

*Grai

Herioa duk hurbildua

Hire ganik.

La musique de ces deux cantiques est aussi ce qui peut se concevoir de plus basque et de plus remarquable selon des maîtres ayant autorité.

Voici, avec neuf vers monorimes à chaque couplet, et en vers inégaux, un genre très étudié, imitant une chanson inspirée par une disette qui sévit sur le pays vers 1825 , disette personnifiée par le poète sous le nom de Betiri Sants. Nous regrettons de ne plus pouvoir trouver nulle part le moindre fragment écrit de ce chant qui avait grande vague dans nos jeunes années:

Betiri-Santsez aspaldi dik

Ez zela deus berri handirik:

Zioten ehortzi zutela bizirik.

Lurpetik ilkirik;

- Ez duk ez irririk; -

Munduz mundu herratua ibilirik ;

Oraikotik ditik Bettirik,

Indarrak hartu, guk utzirik ;

Hor ziagok kargu handitan jarririk (bis).

Ce couplet ne serait pas régulier; il ne répondrait pas aux exigences de la mélodie sur laquelle il doit être chantée, si la rime n'en était unique, et si les quantités syllabiques n'étaient pas exactement ce qu'elles sont là.

Les couplets en vers égaux de huit syllabes et au-dessous, de quelque longueur qu'ils soient, sont assujettis à la même monorimie ; mais ce n'est que dans de rares cas de rythme musical, que la loi de la césure ou de l'hémistiche leur est imposée.

Voici un type de ce genre :

Mehetegiko chakhurra

Zangoz errainez makhurra,

Berga bat luze muthurra, 
Larrutik hurbil hechurra,

Urthe guzian barura

Zeren zen toki chuhurra.

Le poète qui adopte ces mesures-là est tenu d'en observer les règles... S'abstenir vaut mieux que de mal composer.

Dans le paragraphe suivant, nous allons étudier les origines de ces lois prosodiques, et indiquer les types séculaires auxquels on peut les reporter.

\section{Zalduby.}

(Eskualduna 644, 1899-X-13? ; 644, 1899-X-20)

(Suite)

(Voir les n. 611, 613, 616,621, 624, 631 de l'Eskualduna.)

\section{VII}

La poésie, a-t-on dit, est vieille comme l'homme, elle apparaît à l'origine de toutes les littératures : elle a chanté dès l'enfance de tous les peuples; elle chantera tant que durera l'humanité. En chaque race elle a eu elle-même une enfance, et ses règles définitives ne se sont fixées qu'avec le temps sous l'influence de génies plus particulièrement inspirés, inaugurateurs de formes littéraires plus harmoniques.

La poésie basque n'a pas échappé à cette loi : elle se ressent encore des défectuosités de ses évolutions. Comment et que chantait-elle dans les temps anciens? Il est certain que de tout temps les Basques ont chanté : d'aucuns même prétendent que le nom de Cantabres leur fut donné à cause de leurs chants. Mais ils n'écrivaient jamais ni leur histoire ni leur chants. Leur merveilleuse langue leur était peut-être un dépôt trop sacré pour en communiquer par écrit les mystères à d'autres peuples ; ils avaient peut-être aussi à cœur d'être seuls à pouvoir parler et chanter dans l'idiome spécifique de leur race, dont toute lèvre étrangère est encore aujourd'hui si rebelle à reproduire ses accents.

Toutefois, un monument curieux de l'antique poésie euskarienne, et de l'époque des conquêtes romaines, nous a été conservé parmi les épaves littéraires de l'ancienne Cantabrie. De quatorze stances qui purent, vers 1590 , être transcrites d'un manuscrit sur parchemin à peine lisible, voici les trois premières :

Lelo! il Lelo;

Lelo! il Lelo;

Leloa! Zarac

Il Leloa.

Eromaco arotzac

Aloguin eta

Viscaiac daroa

Cansoa.

Octabiano

Munduco jauna, 
Lecobidi

Viscaioa (sic).

Itchassotik

Eta leorrez

Imini deuscu

Molsoa.

Ces quatre couplets de quatre petits vers pourraient bien n'être au fond que deux de deux vers, ou seulement quatre vers rimés distincts. Ignorant sur quel air cela se chantait nous ne saurions nous prononcer. Pour 4 vers complets, la mesure serait sans doute tout aussi irrégulière; mais la rime en est d'une régularité frappante, remarquable surtout pour l'époque à laquelle on fait remonter cette poésie :

Lelo, il Lelo, Lelo, il Lelo ! Leloa zarac, il Leloa!

Eromaco arotzac aloguin eta, Vizcaiac daroa cansoa.

Octabiano munduco jauna, Lecobidi Viscaioa.

Ichassotik eta leorrez, imini deusku molsoa.

A ce texte, qui même pour les Basques actuels a ses obscurités, nous osons, toutefois avec hésitation et réserve, donner dans l'Eskuara d'aujourd'hui l'interprétation suivante :

Lelo ! hil (da) Lelo ! Lelo ! hil (da) Lelo !

Leloa! tzarrak (edo gaichtoak) hil du Leloa!

Erromaco arrotzak alo! (edo achut) egin eta,

Bizkaiak derro (edo errandio) kantoa (irrinzina).

Octabiano munduko jauna da,

Lekobidi da Bizkaikoa.

Itsasotik eta* leihorrez *eto

Ibeni (lasterka jin) zauku (etsai) multzoa.

Du temps d'Octavianus, Auguste, premier empereur des Romains, les Basques rimaient-ils donc déjà avec cette perfection? Si le chant de Lelo est réellement authentique, ce serait le monument le plus ancien de la poésie rimée que nous aient laissé les siècles.

Le savant Huet, évêque d'Avranches vers 1680 et après lui La Harpe, ont pensé que la rime française descend des bardes des Pyrénées; "soit qu'ils en fussent les inventeurs, soit qu'ils l'eussent empruntée aux Maures, avec d'autant plus de vraisemblance que la rime chez les Arabes était de la plus haute antiquité...".

L'assertion de ces deux grands littérateurs ferait déjà, dans ces limites, trop d'honneur aux Basques qui étaient en effet du temps des Maures à peu près les seuls habitants des Pyrénées, et avec qui six cents ans d'alternatives de guerre et de paix avaient mis les Maures en contact. Au surplus, il est généralement reconnu que Maures et Basques se reconnaissaient une certaine communauté d'origine ou quelque ancienne affinité de race, notamment par les Berbères de la Lybie dont la géographie antique est remarquablement euskarienne.

Le rôle saillant, le rôle constant que la rime joue dans la poésie basque, au moins depuis le moyen âge, est incontestable, et son influence sur les langues voisines n'aurait rien d'étonnant. Nous serions tout aussi portés à croire que surtout 
la langue latine, et l'idiome euskarien se seraient pénétrés mutuellement et simultanément, il y a déjà des siècles, de ce goût pour la rime.

Les contemporains de Cicéron, et bien auparavant aussi ceux de Demosthènes, goûtaient fort les désinences pareilles des phrases et des membres de phrases, soit en prose, soit en vers : similiter desinentia. On rimait déjà alors ad voluptatem aurium.

Cicéron lui-même cite avec plaisir un exemple de vers rimés du vieux poète Ennius, chantant les malheurs de Troie :

Haec omnia vidi instammari,

Priamo vi vitam ereptari,

Jovis aram sanguine turbari.

Ces trois vers à la suite avec la même rime riche,...en vérité cela ressemble bien au basque :

Iriongo hiria... la ville d'Ilion. - Les noms basques: Iria, Irion, Iriberri, étaient prononcés par les latins Ilia, Ilion, Illiberi. - Ville, bonne ville, ville neuve.

Iriongo hiria zen erretzen ari

Oi, ikustea bera zein zen izigarri !

Nihor ez zen hargatik eman ihesari

Ez nahiz ukho egin hiri maiteari.

Citons aussi deux vers d'Horace :

Non satis est pulchra esse poëmata, dulcia sunto,

Et quocumque volent animum auditoris agunto.

La rime fourmille dans Ovide et Virgile, surtout dans les hexamètres où le dernier mot se termine par le même son que celui du mot de l'hémistiche. Un chercheur en a compté 15 dans les Bucoliques; 198 dans les Géorgiques et 651 dans l'Enéide : ce qui donne dans Virgile un vers léonin sur quatorze.

Mais c'est surtout à partir du quatrième siècle que la rime pénètre la prose et la poésie latines. Saint Augustin en fit un grand usage dans ses écrits où les consonances sont presque toutes très riches. Les hymnes si nombreux de saint Ambroise abondent d'assonances et de vraies rimes, mais sans méthode uniforme et suivie. Sédulius y met plus de régularité : il a les rimes deux par deux, telles que les a actuellement la versification française.

Peu à peu, dans les hymnes liturgiques, le vers syllabique rimé prédomine sur toutes les délicatesses de la métrique classique, et la langue basque se trouve là dans son élément naturel, goûtant surtout l'effet plus continu de la monorimie inaugurée dans le latin par saint Bernard.

De ce dernier type, adopté préférablement par le génie populaire de nos pays, nous donnons ici quelques exemples.

Voici d'abord les premières strophes de deux hymnes de saint Bernard sur le saint Nom de Jésus : 
AD MATUTINUM

Jesu, * dulcis memoria,

Dans* vera cordis gaudia :

Sed super mel et omnia, *

Ejus dulcis praesentia.
AD PRIMAM

*Jesu dulcis... Cum Maria diluculo,

*Dan Jesum quaeram in tumulo,

*omnia. Cordis clamore quaerulo,

Mense quaeram non oculo.

En basque cela pourrait librement se traduire ainsi :

Jesusen izen eztia,

Bihotz loriagarria.

Zure gana dut lehia;

Zurekin naiz bat nahia.
Goazen hobira goizetik, Madalenaren ondotik, Bihotzak bozik, gogotik, Jesu piztu da ingoitik.

Saint Bonaventure nous offre un modèle parfait de nos chants en treize sy]labes 7-6 qui sont les plus usités:

Philomena praevia temporis amoeni

Quae recessum nuntias imbris atque caeni.

Dum mulcescis animos tuo cantu leni

Ave prudentissima, ad me quaeso, veni.

Le 8-6 à rimes croisées du Pange lingua, de saint Thomas d'Aquin, n'a que très peu de similaires en basque avec cette complication de la double rime :

Pange lingua, gloriosi

Corporis mysterium

Sanguinisque pretiosi* * protiosi

Quem* in mundi pretium *quen

Fructus ventris generosi

Rex effudit gentium.
Jakintsunen erranetan

Behar bada sinheste

"Ederrena mintzaietan

basque) "Eskuara da diote."

Eskualdunek arrotzetan

Hortaz ohore dute.

Ce 8-6 ordinairement est traité en vers de 15 pieds divisés en hémistiches dont les pairs seuls sont rimés :

Munduko urgaindiekin gabiltzanak borrokan, (bis)

Eskualdunak berma gaiten Fedearen harrokan,

Iduk gaitzatzu, Maria, leheneko herronkan.

Mais plus souvent les strophes de pareils vers se composent de quatre vers :

Zorigaitzez egun batez zortheak atzemanik

Nere burhaso zaharrak nigarretan utzirik

Soldadu nintzela bada harmetan ezarririk

Urrun ereman ninduten, nere Eskual-herritik.

Le jeu des rimes aux croisements entrelacés, tel que le pratiquait Adam de Saint Victor dans ses proses délicieuses, a été aussi de rares fois, mais imparfaitement, imité par les chants basques :

Martyris egregii,

Triomphos Vicentii

Celebrat Ecclesia,

Qui certanti praefuit,

Vires arma praebuit,

Regi laus et gloria.
Nafartarren arraza

Hila ala lo datza?

$\mathrm{Ez}$ dut endelgatzen.

Belzunze bizcondia

Hain gerlari handia,

Ez baitzaut mintzatzen ;

Hori zaut gaitzitzen. 
Voici un autre exemple :

O Virgo sapiens!

$O$ Verbo vagiens !

Zure gana, Jauna,

O Masestas humilis!

Nos juva, nos rege,

Nos verbo protege,

Nobis carne similis.

Ene jabe ona,

Itzultzen naiz bihotzez :

$\mathrm{Zu}$ gabe lurrean

Bethi gaizkipean

Ibili naiz, ibili naiz...trebes.

(A suivre)

\section{Zalduby}

\section{Xabier ALTZIBAR}

U.P.V 